for

\title{
Template-less Synthesis of Coded Au Nanowires
}

Xuesong Wu, Hongyan Li, Weiyu Wang, Dongmeng Su, Xi Wang, Xiaolin Tao, Yawen

Wang* and Hongyu Chen*

Institute of Advanced Synthesis, and School of Chemistry and Molecular Engineering,

Jiangsu National Synergetic Innovation Center for Advanced Materials, Nanjing Tech

University, Nanjing 211816, People's Republic of China

\section{Contents:}

1. Materials and Instruments

2. Experimental Section

3. Figure S1: Large area view SEM image of the coded Au nanowire bundles.

4. Figure S2: Measurement of the open-circuit potential.

5. Figure S3: No Au deposited was observed at extreme high potential.

6. Figure S4: Diameter distribution of ultrathin Au nanowires generated within high potential range.

7. Figure S5: Mixture of thin nanowires and non-uniform bundles generated at alternate potential on an Au seed adsorbed-substrate.

8. Figure S6: Sparse nanowire bundles formed at high potential on a seedless substrate.

9. Figure S7: Small dendritic-like Au nanostructures generated on a seedless substrate at low potential.

10. Figure S8: Compare with the chemical synthesis, electrochemical deposition significantly reduced the chance of the homogeneous nucleation at a fast reduction rate.

11. Figure S9: Screen of the low potential threshold for generating coded Au nanowire in MPS method. 
12. Figure S10: Caked mass formed at the tips of long Au nanowires.

13. Figure S11: Ultralong nanowires with length more than $50 \mu \mathrm{m}$.

14. Figure S12: Surface resistance measurement of the substrates with different deposition time.

15. Figure S13: Au nanowire bundles containing thick branched nanowires generated by alternating potential at $0.5 \mathrm{~s}$ intervals.

16. Figure S14: Large area view SEM images of the coded Au nanowire emulating the Morse code. 


\section{Materials and Instruments}

Materials: Hydrogen tetrachloroaurate(III) hydrate (HAuCl4 • 3H2O, 99.9\%) and 4-mercaptobenzoic acid(4-MBA, 99\%) were purchased from Sigma-Aldrich. 3-aminopropyl-triethoxysilane (APTES, 99\%) was purchased from aladdin. Trisodium citrate dihydrate $(\mathrm{Na} 3 \mathrm{C} 6 \mathrm{H} 5 \mathrm{O} 7 \cdot 2 \mathrm{H} 2 \mathrm{O}, 99 \%)$ was purchased from Alfa-Aesar. Deionized water (resistivity $>18 \mathrm{M} \cdot \mathrm{cm}-1$ ) and ethanol (AR) were used to prepare all solutions and electrolyte. All these chemicals were used as received. 100-p type Si wafer was purchased from Electron Microscopy China.

Instruments: Field emission scanning electron microscopy (SEM) images were collected on a FEI Quanta 250 FEG model operated at $30 \mathrm{kV}$. Transmission electron microscopy (TEM) images were collected from a Talos L120C model operated at $120 \mathrm{kV}$. Electrochemical workstation dates were collected on $\mathrm{CH}$ instruments model $1040 \mathrm{C}$ and $650 \mathrm{E}$.

\section{Experimental Section}

Ultrasonic cleaning of $\mathrm{Si}$ wafer: A cut $\mathrm{Si}$ wafer (about $1.3 \mathrm{~cm} 2$ ) was first rinsed, and then ultrasonicated in acetone, ethanol and deionized water for $30 \mathrm{~s}$ respectively.

Hydrophilicity treatment and Au nanoparticles seed decoration on Si wafer: The cleaned $\mathrm{Si}$ wafer was treated with $\mathrm{O} 2$ plasma for $10 \mathrm{~min}$ in order to improve its hydrophilicity ${ }^{1}$. Amino group was introduced by immersing the wafer in water/ethanol $(1: 1, \mathrm{v} / \mathrm{v})$ diluted APTES solution $(5 \mathrm{mM})$ for $0.5 \mathrm{~h}$. Subsequently, the wafer was rinsed twice with water to remove the residual APTES and then soaked in citrate-stabilized Au nanoparticle (3-5 nm) solution for $0.5 \mathrm{~h}$ to adsorb the Au seeds.

Electrochemical deposition of Au nanowires on Si wafer: The Au seeds-adsorbed Si wafer was directly used as the working electrode in a three-electrode system. It was partially immersed in a waterethanol mixture $(2: 1, \mathrm{v} / \mathrm{v})$ electrolyte containing $1.17 \mathrm{mM}$ of $\mathrm{HAuCl} 4$ as Au precursor and $0.20 \mathrm{mM}$ of 4-MBA as capping ligand. No extra supporting electrolyte was added. A $25 \mathrm{~mL}$ three-neck round-bottom flask was used as the electrochemical cell. The electrodeposition was carried at room temperature without stirring. The applied electrochemical method and parameters are determined by the specific experiments. During the deposition, the top part of $\mathrm{Si}$ wafer immersed in the solution gradually turned black, signifying the formation of $\mathrm{Au}$ nanostructures. The yellow color of electrolyte remained unchanged. After deposition, the wafer was retrieved and stored in ethanol before further characterization. 
Synthesis of 3-5 nm Au seed solution: The citrate-stabilized Au nanoparticle seed with a diameter of 3-5 nm was synthesized based on the reported method. ${ }^{2}$

Preparation of SEM sample: The as-prepared wafer was dried in the air and directly subjected to SEM characterization.

Preparation of TEM samples: The Au nanowire was re-dispersed in ethanol by sonication, concentrated and washed multiple times before dripping on the TEM grid. The TEM grid was treated with $\mathrm{O} 2$ plasma for $60 \mathrm{~s}$ to render its surface hydrophilic.

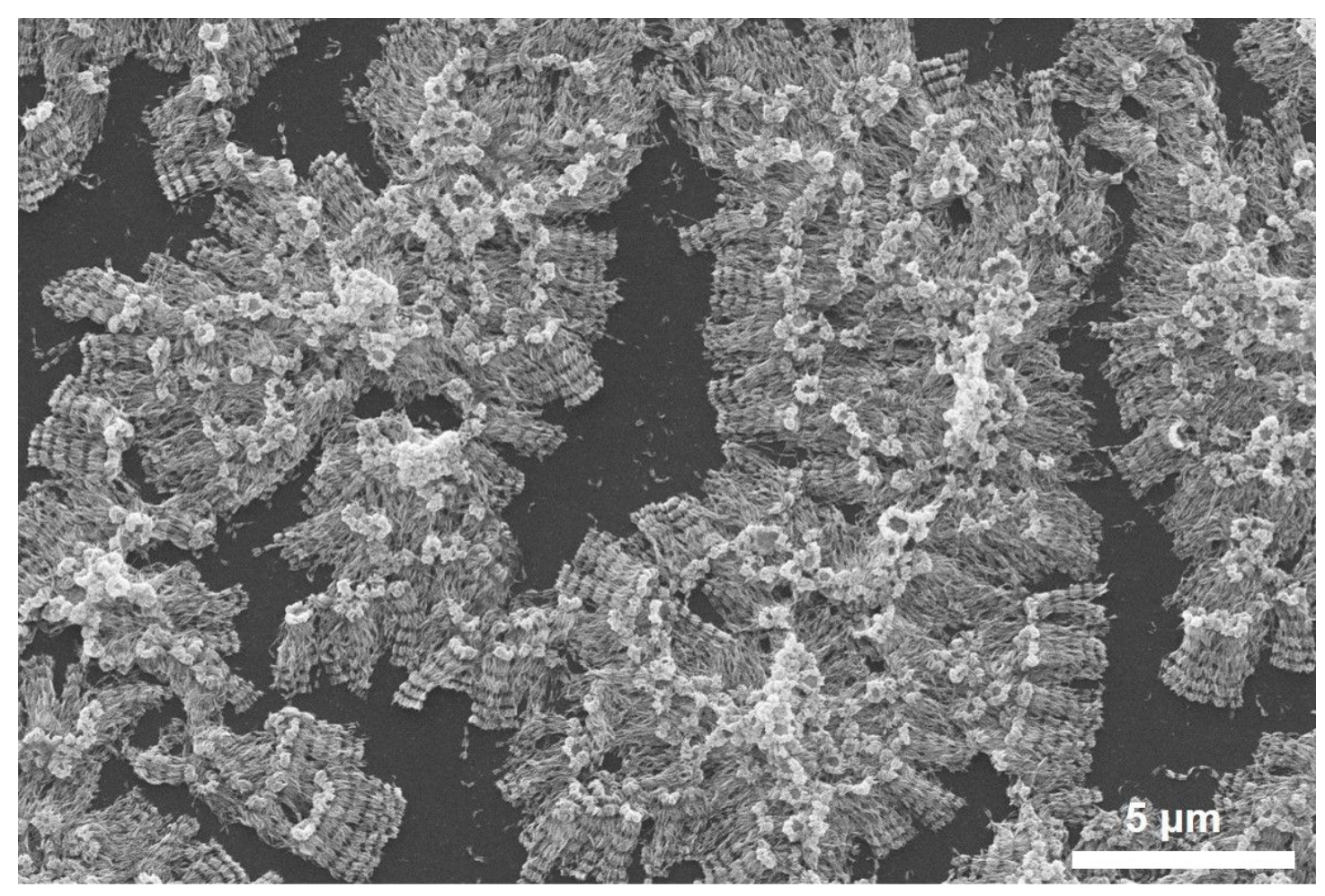

Figure S1. Low magnification SEM image of the coded Au nanowire bundles.

The "caps" at the top of each bundles are the initial thick segment caused by the expansion of the active sites at the beginning of the growth. 


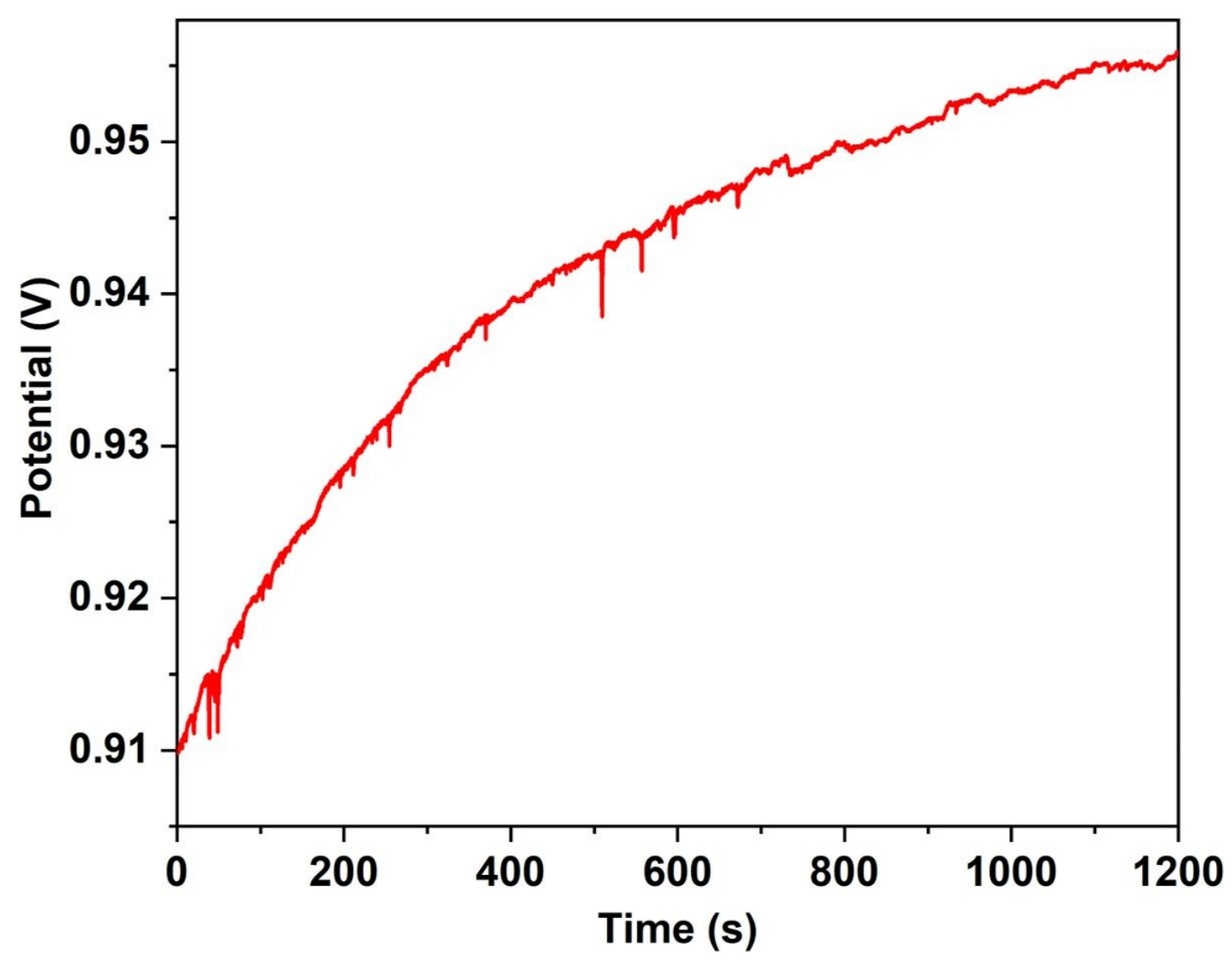

Figure S2. The open-circuit potential was stabled at around $0.955 \mathrm{~V}$ after 20 min measurement. 
Figure S3. No Au deposited was observed after 10 min deposition at $0.95 \mathrm{~V}$ (vs. $\mathrm{Ag} / \mathrm{AgCl}$ ).

The small nanoparticles are the pre-absorbed 3-5 nm Au seed.

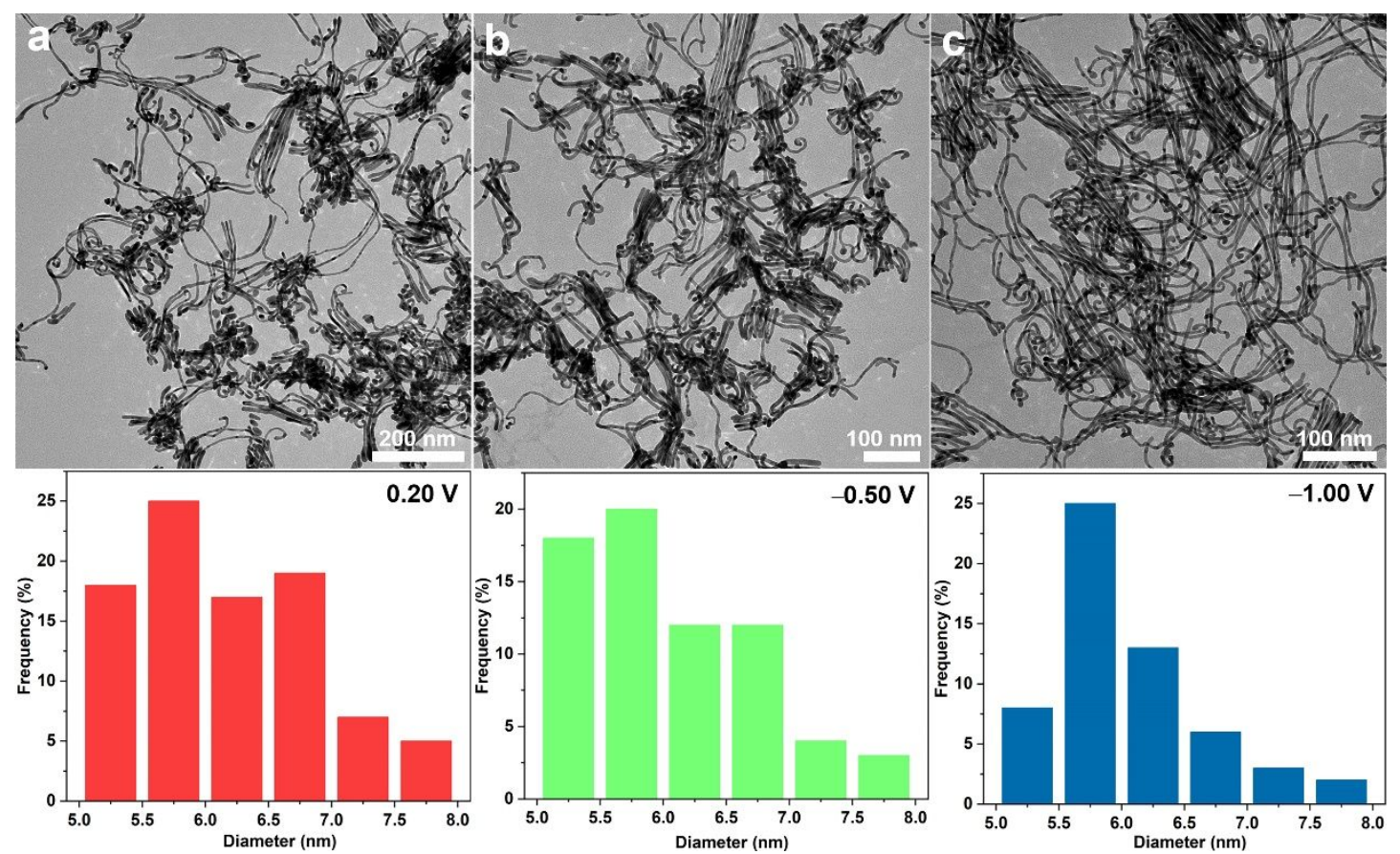

Figure S4. Diameter distribution of ultrathin Au nanowires generated at : (a) $0.20 \mathrm{~V}$; (b) $-0.50 \mathrm{~V}$; (c) $-1.00 \mathrm{~V}$ vs. $\mathrm{Ag} / \mathrm{AgCl}$ 


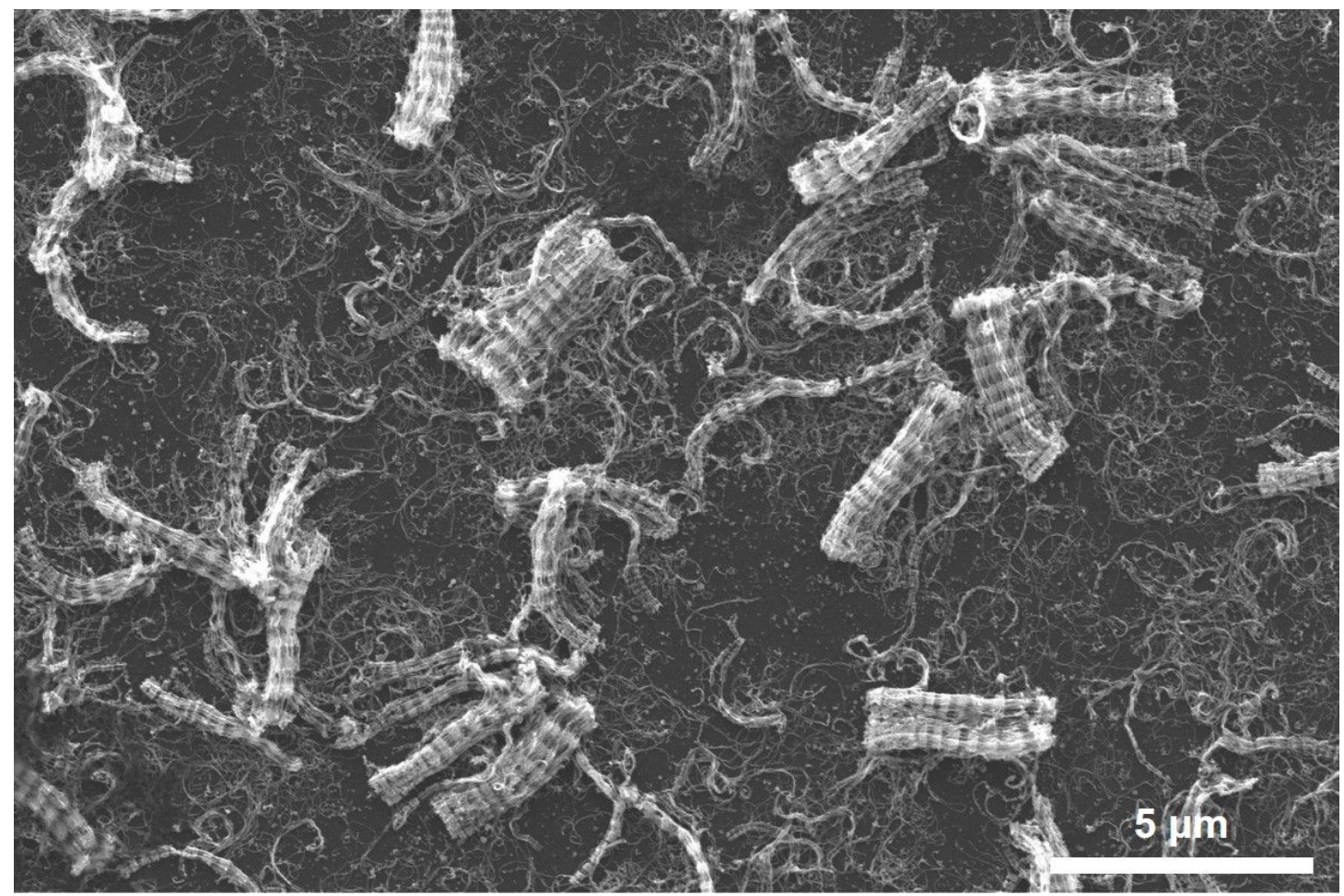

Figure S5. On an Au seed adsorbed-wafer, mixture of thin nanowires and non-uniform bundles generated at alternate potential between -5.00 and $-0.50 \mathrm{~V}$ for 12 cycles. The duration for each step was $15 \mathrm{~s}$. In this case, sequent growth (thin nanowire) and heterogeneous nucleation (bundle) happened simultaneously. 


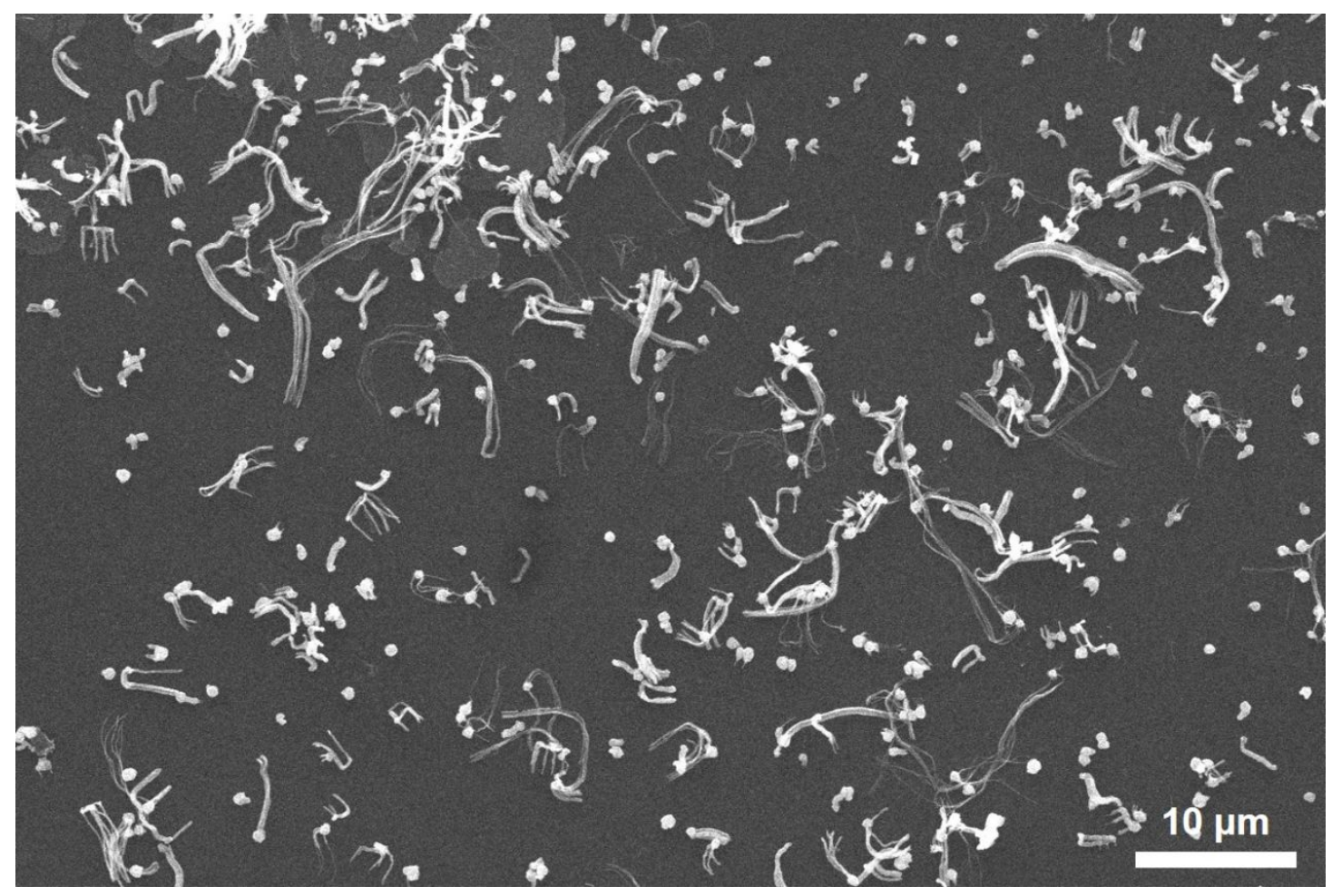

Figure S6. Sparse nanowire bundles formed after $5 \mathrm{~min}$ reduction at $-0.50 \mathrm{~V}$ (vs. $\mathrm{Ag} / \mathrm{AgCl})$.

Without pre-adsorbed seeds, the slow reduction rate at $-0.50 \mathrm{~V}$ could create only a few heterogeneous nucleation sites. Once these nuclei formed, they would facilitate the following Au deposition and the heterogeneous nucleation nearby would be inhibited. Meanwhile, the amount of Au to be deposited on each nucleus was in great excess. As a result, the active surface on each growth sites would expand to form of nanowire bundles. 


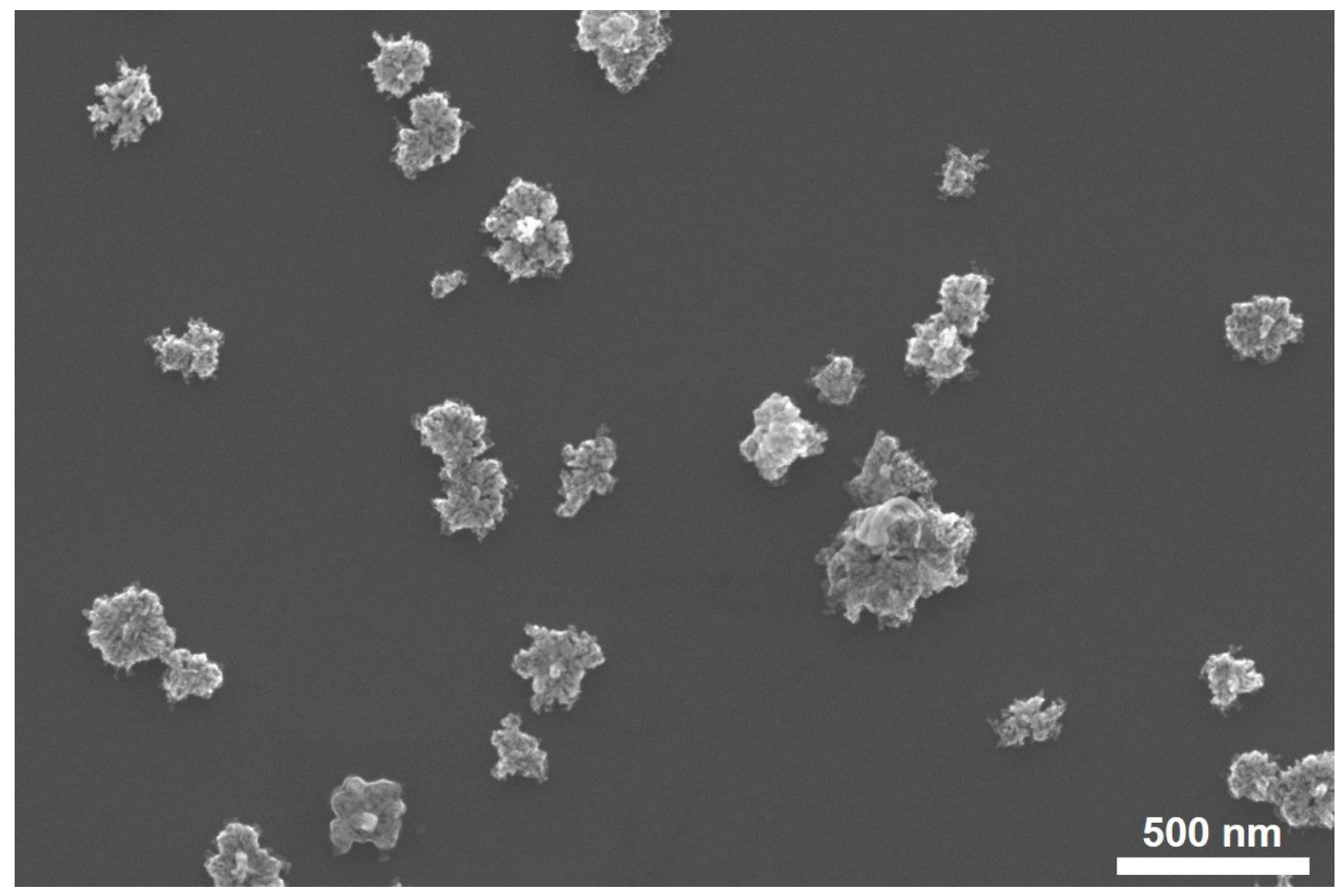

Figure S7. Small dendritic-like Au nanostructures generated on a seedless substrate after 30 s reduction at $-5.00 \mathrm{~V}$ vs. $\mathrm{Ag} / \mathrm{AgCl}$. 


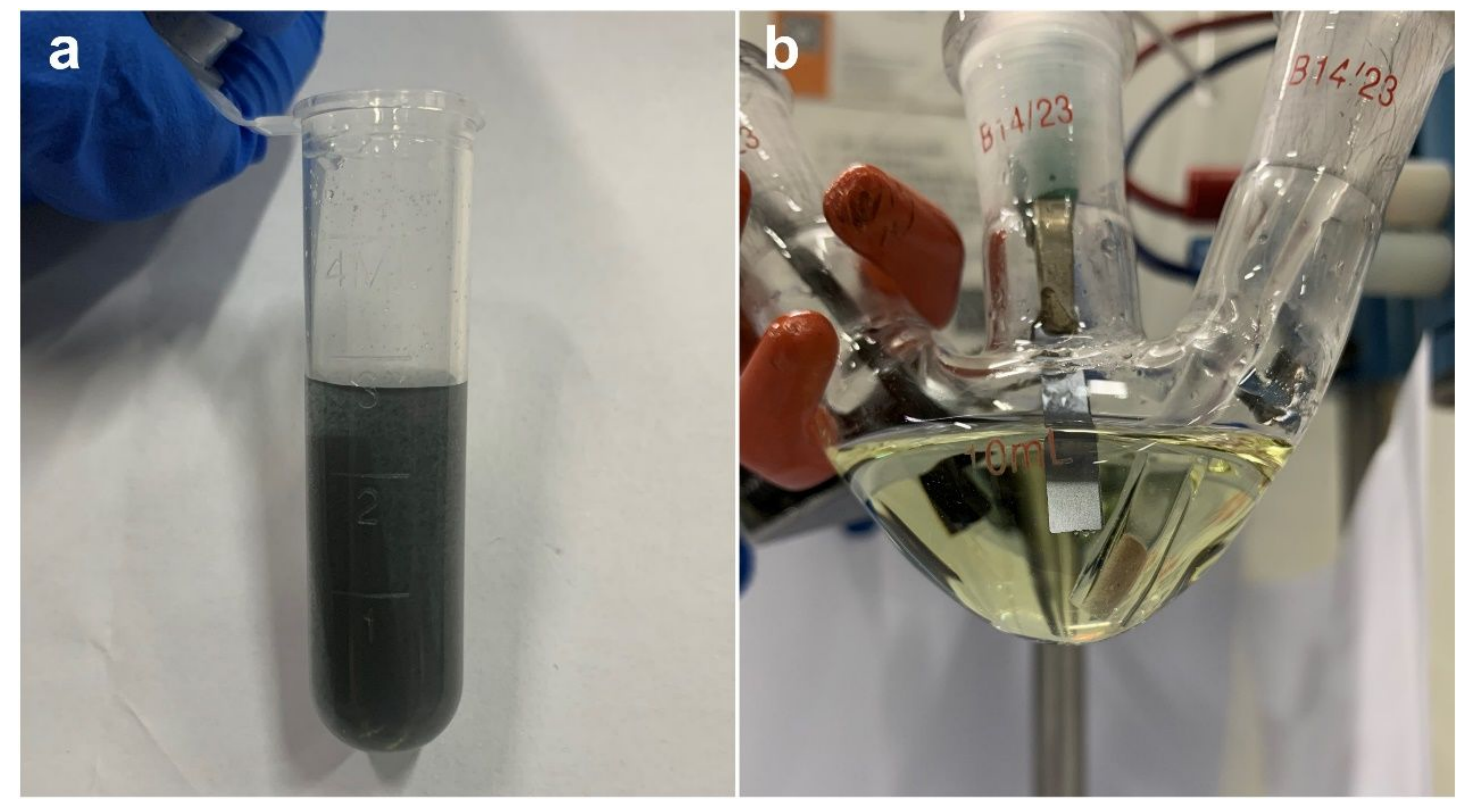

Figure S8. (a) In chemical synthesis, increase the concentration of the reductant (L-ascorbic acid) would significantly speed up the reduction. But the nanowire growth would be uncontrollable due to the severe homogeneous nucleation. For instance, homogeneous nucleation occurred immediately after adding 16.4 $\mathrm{mM}$ (4 times of the normal concentration) of L-ascorbic acid, as indicated by the color of the growth solution and the precipitation. (b) In electrochemical deposition, no homogeneous nucleation in the electrolyte was observed after reducing at $-6.25 \mathrm{~V}$ (vs. $\mathrm{Ag} / \mathrm{AgCl})$ for $10 \mathrm{~min}$. 



Figure S9. Screen of the low potential threshold for generating coded Au nanowire in MPS method. SEM image of the segmental nanowires obtained by alternating the applied potential at $30 \mathrm{~s}$ intervals for 6 cycles between $-0.50 \mathrm{~V}$ and (a) $-1.30 \mathrm{~V}$; (b) $-1.60 \mathrm{~V}$; (c) $-1.90 \mathrm{~V}$ vs. $\mathrm{Ag} / \mathrm{AgCl}$. 


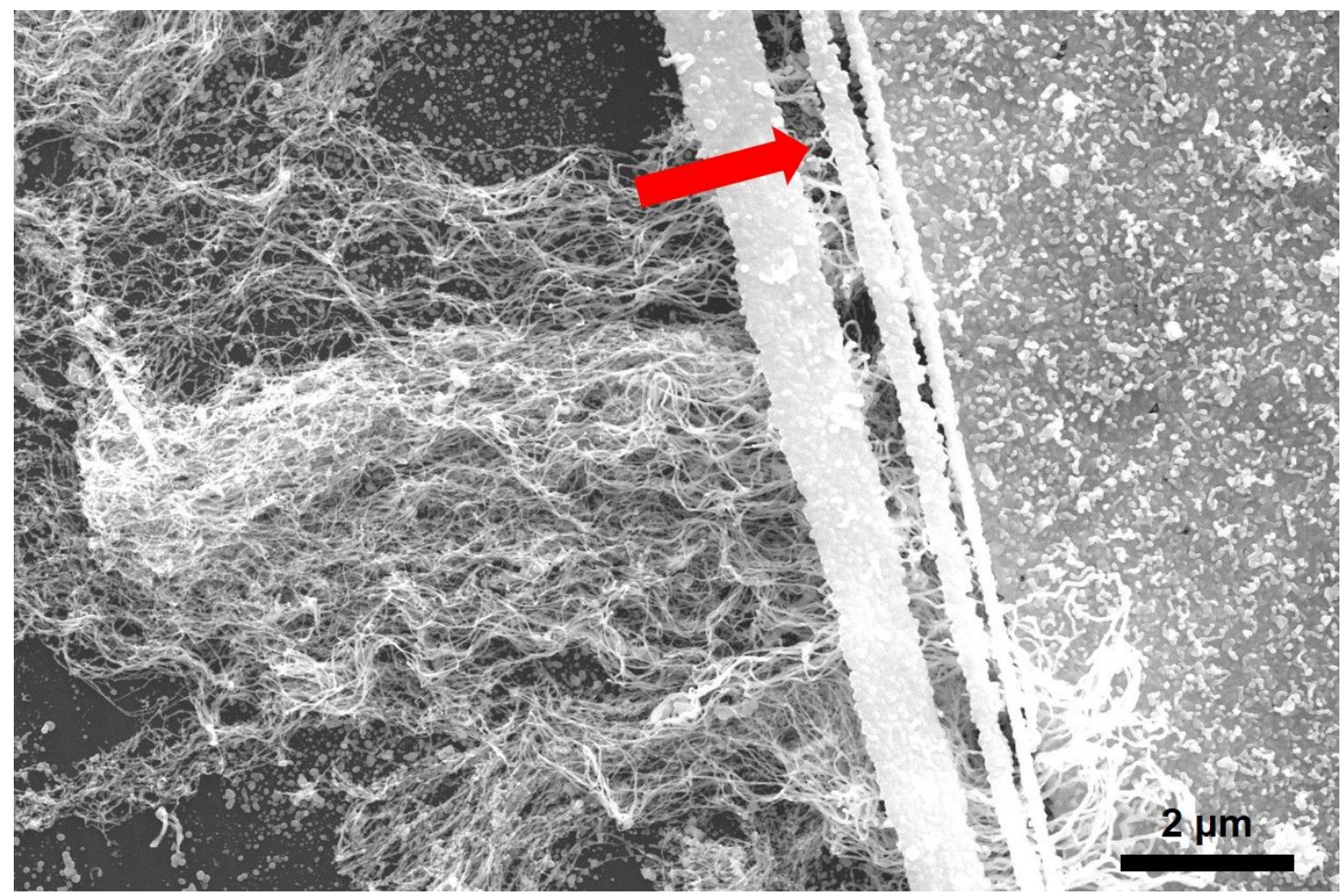

Figure S10. Au nanowires longer than $10 \mu \mathrm{m}$ obtained by deposition at $-0.10 \mathrm{~V}$ for $1 \mathrm{~h}$. Significant caked mass on the nanowire tips could be observed (red arrow).

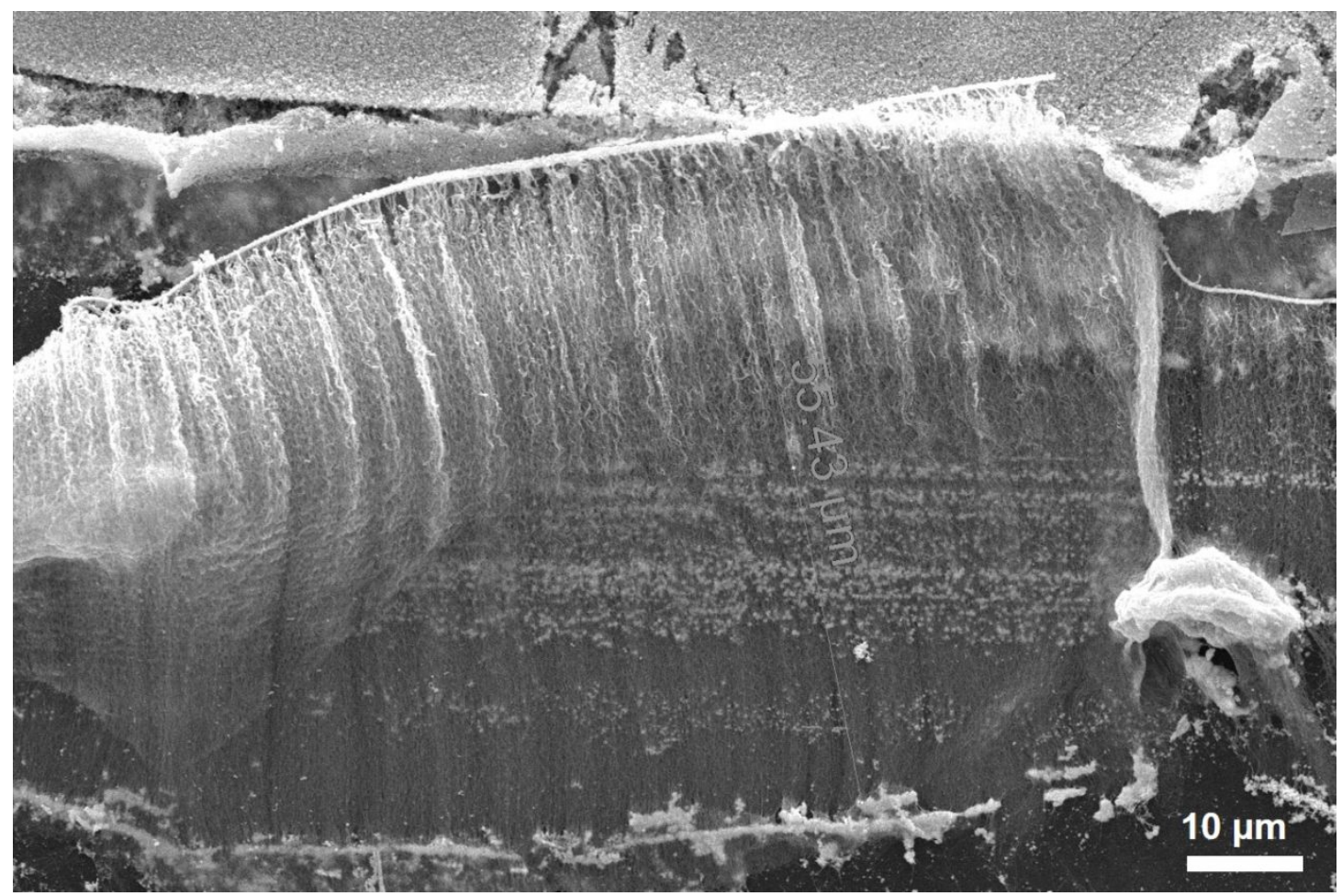

Figure S11. SEM image of a very long nanowire bundle obtained after $4 \mathrm{~h}$ deposition at $-0.10 \mathrm{~V}$. 


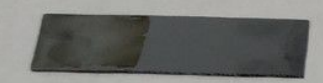

$10 \mathrm{~min}$

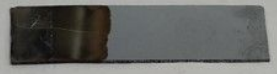

$30 \mathrm{~min}$

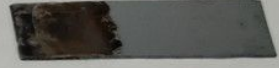

$90 \mathrm{~min}$

Figure S12. Digital graph of the wafer electrodes obtained after 10, 30 and 90 min deposition of Au nanowires at $-0.50 \mathrm{~V}$. The sheet resistance of these samples measured by four-point probe method was $6.96 \times 10^{3}, 16.34$, and $2.45 \Omega \mathrm{sq}^{-1}$, respectively. 


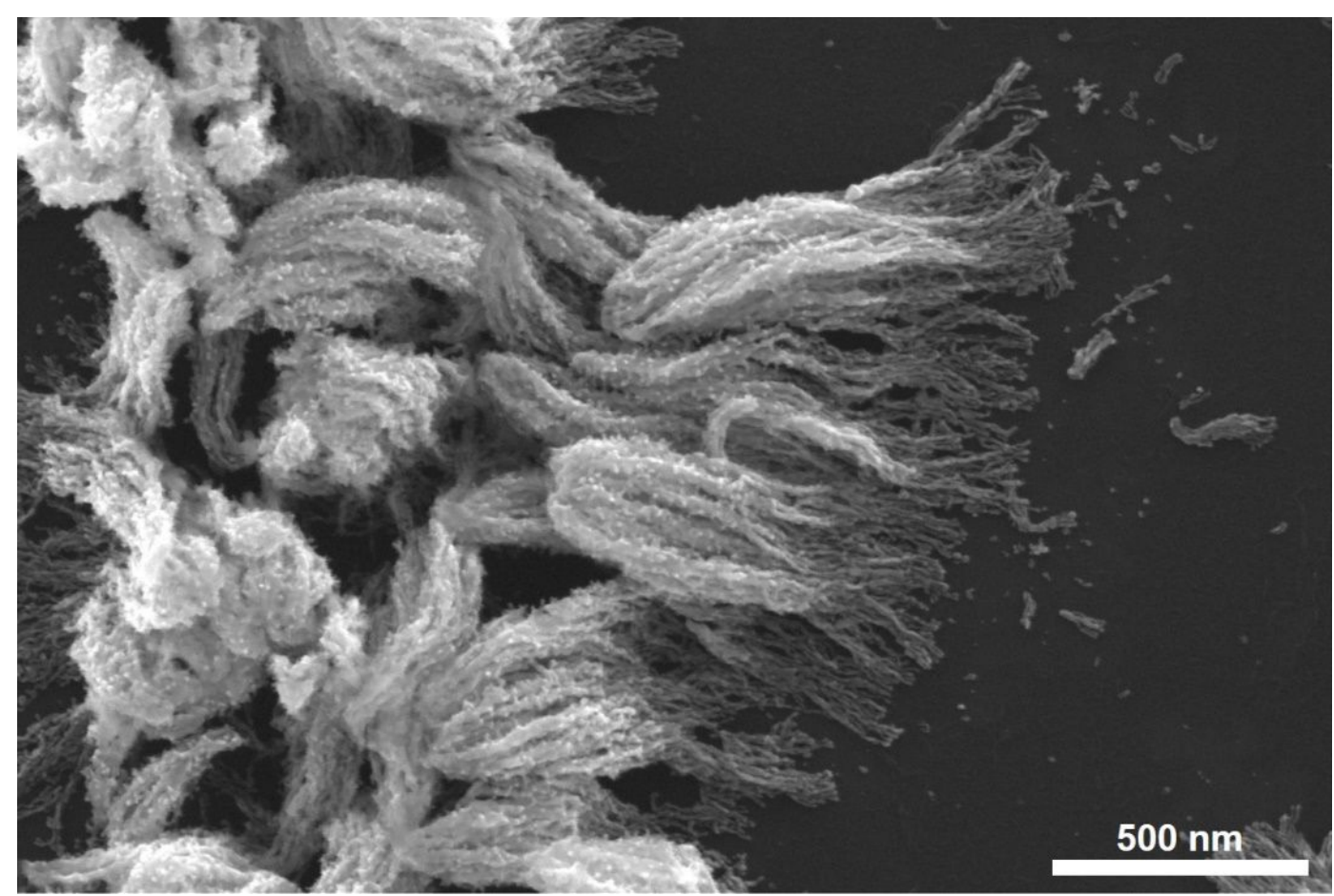

Figure S13. Au nanowire bundles containing thick branched nanowires generated by alternating potential between $-5.00 \mathrm{~V}$ and $-0.50 \mathrm{~V}$ at $0.5 \mathrm{~s}$ interval for 360 cycles. 

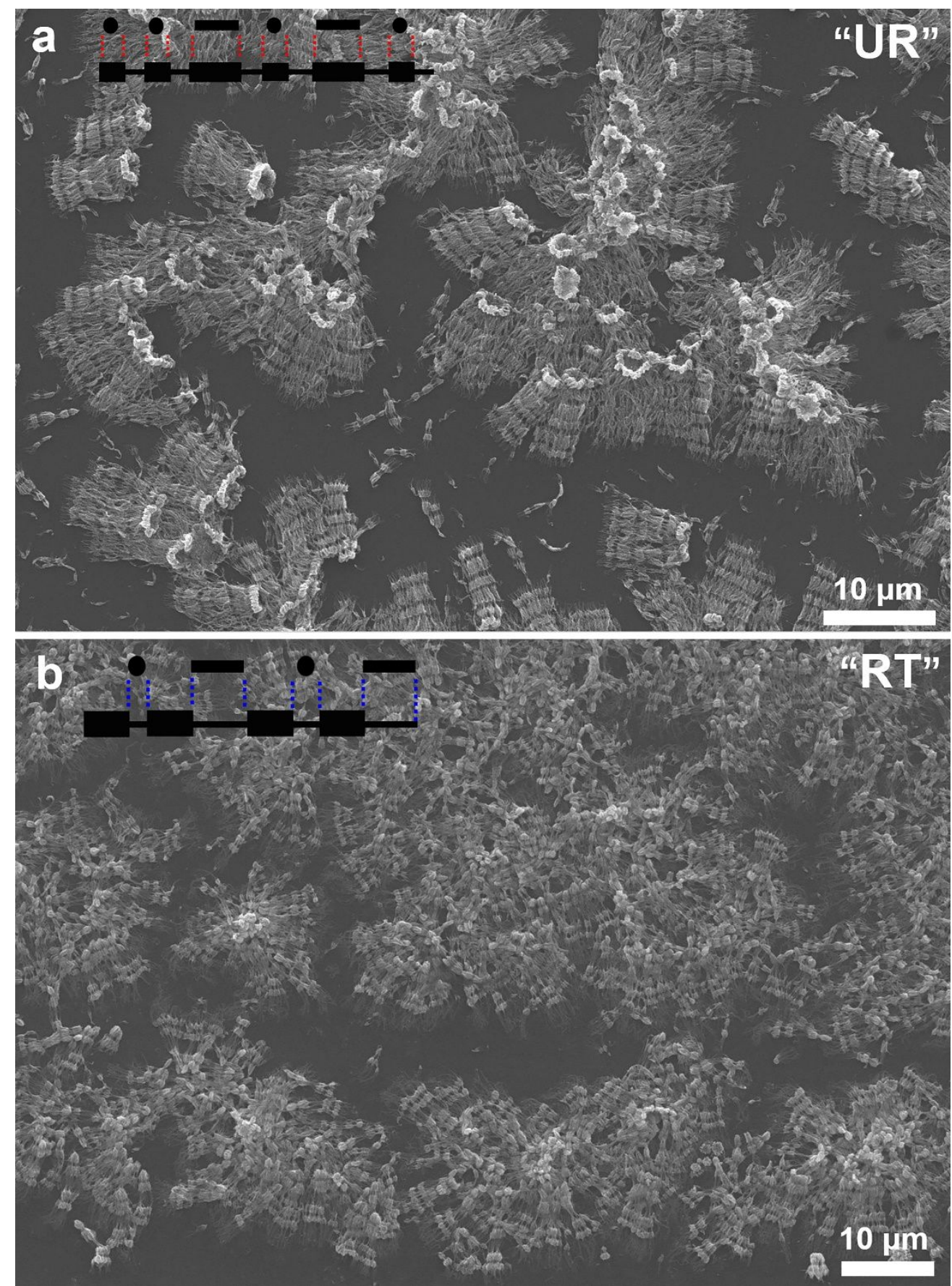

Figure S14. Large area view of the coded Au nanowire: (a) letters combination "UR"; (b) letters combination "RT".

Note that the deposition time of the last thin segment (dash) was extended from 30 to $70 \mathrm{~s}$ to improve the contrast between the dot. 


\section{Reference}

(1) R. Bardhan, S. Mukherjee, N. A. Mirin, S. D. Levit, P. Nordlander, N. J. Halas, The Journal of Physical Chemistry C 2010, 114, 7378-7383.

(2) G. Chen, Y. Wang, M. Yang, J. Xu, S. J. Goh, M. Pan, H. Chen, Journal of the American Chemical Society 2010, 132, 3644-3645. 